\title{
A Multi-Phase-Field Anisotropic Damage-Plasticity Model for Crystalline Rocks
}

\author{
SeonHong $\mathrm{Na}$ (D) and WaiChing Sun ${ }^{(\varpi)}$ (D) \\ Columbia University, New York, USA \\ wsun@columbia.edu
}

\begin{abstract}
Many engineering applications, such as geological disposal of nuclear waste, require reliable predictions on the thermo-hydro-mechanical responses of porous media exposed to extreme environments. This presentation will discuss the relevant modeling techniques designed specifically for such environmental conditions. In particular, we will provide an overview of the coupling method of crystal plasticity and multi-phase-field model designed to replicate the thermal- and ratedependent damage-plasticity of crystalline rock. Special emphasis is placed on capturing the intrinsic anisotropy of salt grain in $3 \mathrm{D}$ with respect to damage behavior and plastic flow by incorporating the crystallographic information of salt.
\end{abstract}

Keywords: Crystal plasticity $\cdot$ Damage-Plasticity $\cdot$ Multi-Phase-Field model Salt

\section{Introduction}

The permanent geological disposal of nuclear waste in salt formations is one of the most feasible options to meet the increasing demand for nuclear waste storage. The reason for the popularity of salt formations is attributed to the desirable thermo-hydro-mechanical characteristics of the salt, i.e., the combinations of low permeability (practically impermeable), high thermal conductivity, relatively chemically inactive, high heat resistance, geological stability, and relatively ductile responses compared to other possible host materials (e.g., shale and granite).

In this work, we present a nonlocal damage-plasticity model designed to capture the anisotropic damage-plasticity of salt at the single-grain micromechanical level in a threedimensional setting. Instead of capturing the anisotropy of the damage-plasticity responses in a purely phenomenological approach, we will introduce a microstructuralaware continuum model for crystalline rock salt. In particular, we will consider salt as a crystalline material with the slip system which dictates the rate-dependent plastic flow. Meanwhile, the anisotropy of fracture behavior will be captured by a multi-phase-field model in which each phase field is assumed to be associated with the orientation of each slip system. 


\section{Method}

In this work, the salt grain is idealized as pure and without any defect or brine inclusion. As such, the work done on the salt grain may lead to increase in the elastic strain energy or can be dissipated via crack growth or plastic flow. Previously, small and finite strain models that couple phase field fracture and cap-plasticity have been proposed to capture the brittle-ductile transition in isotropic materials [1, 2, 6]. Meanwhile, relationship between effective critical energy release rate and the spatial heterogeneity is explored in Na et al. [4]. In addition, the coupling of crystal plasticity and multi-phase-field models for crystalline materials have been reported in $\mathrm{Na}$ and Sun [3]. In this work, the model reported in $\mathrm{Na}$ and Sun [3] is implemented and simulated in a 3D setting. To couple the nonlocal damage, the phase field model with a crystal plasticity model that is iterative in a local return mapping is used with a non-iterative operator-split algorithm. A very small incremental step is adopted to maintain the equilibrium [1, 3, 5]. The governing equations discretized by a standard Galerkin finite element with equal-order basis functions are the balance of linear momentum, balance of energy and the micro-force balance with the displacement (3-dimensional vector, $\boldsymbol{u}$ ), temperature ( $\operatorname{single~scalar,} \theta$ ) and the multiple phase fields (multiple scalars, $\underline{d}$ denotes a set of $d_{i}$ where the value of $i$ is consistent with the number of slip systems of salt grain) as the nodal unknown, i.e.,

\section{Balance of Linear Momentum:}

$$
\nabla \cdot \sigma+b=0 \text { with } \sigma=\frac{\partial \hat{\psi}}{\partial \epsilon^{e}}=g(\underline{d}) \hat{\sigma},
$$

where $\hat{\boldsymbol{\sigma}}=\mathbb{C}^{e}: \boldsymbol{\epsilon}^{e}-3 \alpha K\left(\theta-\theta_{0}\right) \mathbf{1}$ and $g(\underline{d})=(1-k) \prod\left(1-d_{i}\right)^{2}+k$. Here $\mathbb{C}^{e}$ denotes the fourth-order elasticity tensor; $\alpha$ is the thermal expansion coefficient; $K$ is the bulk modulus, $\theta_{0}$ indicates a fixed reference temperature, and 1 is the second-order identity tensor. The degradation function $g(\underline{d})$ for the multi-phase-field has been chosen such that $g^{\prime}\left(d_{i}=1\right)=0$. The small parameter $k \ll 1$ is introduced for maintaining the well-posedness of the problem.

\section{Balance of Energy:}

$$
\begin{aligned}
& c_{v} \dot{\theta}=\left[D_{\text {mech }}-H_{\theta}\right]-\nabla \cdot \boldsymbol{q}+r_{\theta} \\
& \text { with } \underbrace{\sum_{\alpha}\left(\pi^{\alpha} \dot{\gamma}^{\alpha}+g^{\alpha} \dot{s}^{\alpha}\right)+\theta\left(\frac{\partial^{2} \hat{\psi}}{\partial \theta \partial \boldsymbol{\epsilon}^{e}}: \boldsymbol{\epsilon}^{e}\right)}_{\mathrm{D}_{\text {mech }}-\mathrm{H}_{\theta}} .
\end{aligned}
$$

Here $c_{v}$ denotes the specific heat per unit volume at constant deformation; $D_{\text {mech }}$ is the contribution to dissipation due to pure mechanical load and/or thermal flow; $H_{\theta}$ is the non-dissipative (latent) thermoelastic structural heat or cooling. $\boldsymbol{q}$ is the heat flux vector and $r_{\theta}$ indicates the heat source. $\pi^{\alpha}$ and $g^{\alpha}$ are thermodynamic forces powerconjugate to a local plastic slip $\gamma^{\alpha}$ and a local scalar measure related to the plastic slip accumulation for each slip system $s^{\alpha}$, respectively. 


\section{Balance of Micro-forces for Each Crack Phase $d_{i}$ :}

$$
\begin{aligned}
& 2\left(1-d_{i}\right) \mathcal{H}_{i}+\frac{G_{c}}{l} d_{i}+G_{c} l \nabla \cdot\left(\omega_{i} \cdot \nabla d_{i}\right)=0 \\
& \text { with } \mathcal{H}_{i}=\max _{\tau \in[0, t]}\left\{\prod_{j \neq i}\left(1-d_{j}\right)^{2}\left[\omega_{+}^{e}+\omega^{p}-\omega_{0}^{p}\right]\right\},
\end{aligned}
$$

where $G_{c}$ is the fracture energy or the energy release rate for each slip system. $\mathcal{H}_{i}$ is the strain-history functional that governs the evolution of the irreversible crack propagation. $l$ indicates a length scale that controls the width of the smooth approximation of the crack, which is assumed to be larger than the mesh size. $\omega_{i}$ is a second-order structural tensor associated with the slip plane $i$. $\omega_{+}^{e}$ denotes the thermoelastic strain, and $\omega^{p}$ is the plastic work coming from the hardening contribution. $\omega_{0}^{p}$ is introduced to control the plastic deformation in ductile fracture (cf. Na and Sun [3]).

\section{Results and Future Directions}

We conducted three uniaxial compression test simulations on the domain of the $0.4 \times$ $0.4 \times 1.0 \mathrm{~mm}$ and compared of the same materials but of different crystallographic orientations. The Euler Angles, in terms of degree, are $(0,0),(30,10)$ and $(45,0)$, respectively. A pair of incremental displacement is applied on the top of the $3 \mathrm{D}$ domain to introduce the uniaxial compression. The resultant damage (as illustrated by the combined phase field, $d_{\text {combined }}=\sum_{i} d_{i}-\sum_{i \neq j} d_{i} d_{j}+\sum_{i \neq j \neq k} d_{i} d_{j} d_{k}-\prod_{i} d_{i}$ ) and the plastic strain are shown in Figs. 1 and 2 accordingly.
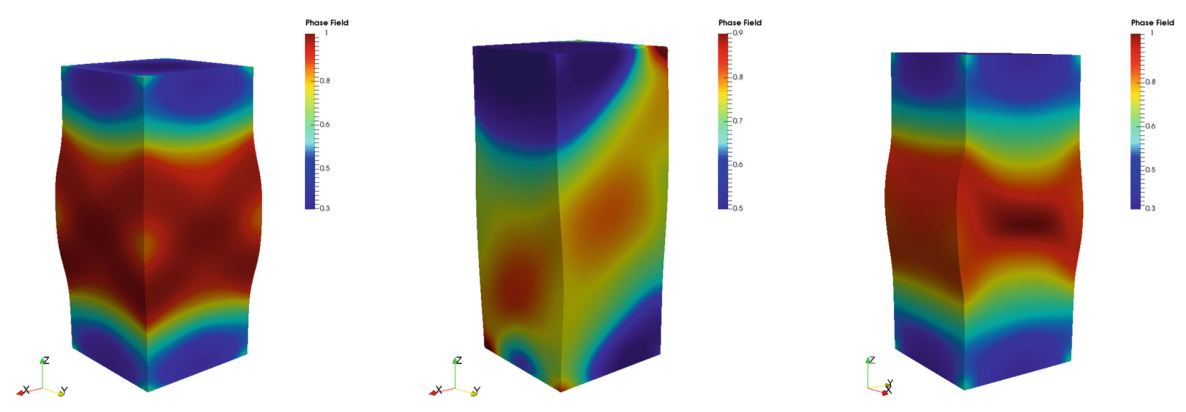

Fig. 1. The resultant damage of uniaxial compression test in 3D domain is described. Fixing the boundary conditions, three sets of different Euler Angles are selected (from the left to right: $(0$, $0),(30,10)$ and $(45,0))$ to represent the anisotropic damage behavior. 

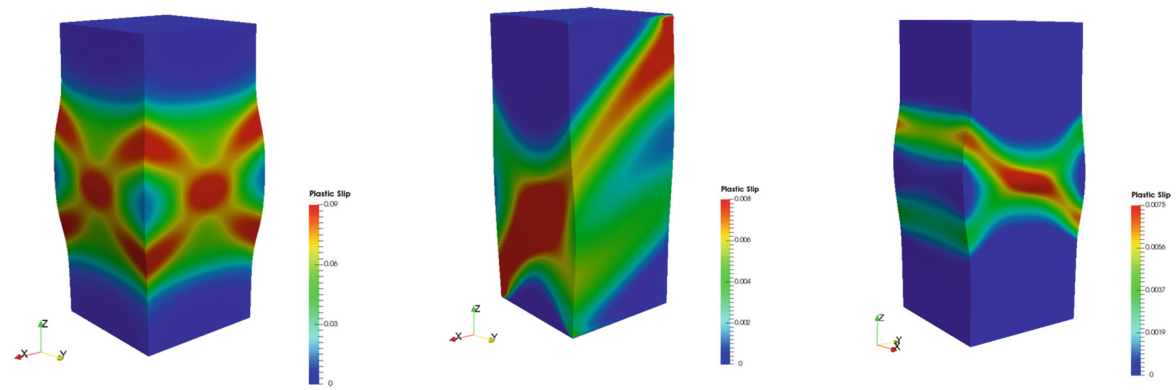

Fig. 2. The resultant plastic behavior of uniaxial compression tests in $3 \mathrm{D}$ domain is depicted. As in Fig. 1, three sets of Euler Angles are used from the left to right: $(0,0),(30,10)$, and $(45,0)$, respectively.

These distinct damage and plastic strain clearly indicate that both the damage and the plastic responses are indeed anisotropic. More importantly, the induced anisotropy is replicated simply by incorporating the orientation of the slip system. Currently, the research group is working on a few new directions, including but not limited to incorporating this model for poly-crystalline materials and for field-scale simulations via a recursive homogenization approach as in Wang and Sun [7] or a concurrent coupling approach, and introducing geometrical nonlinearity.

\section{References}

1. Choo, J., Sun, W.C.: Coupled phase-field and plasticity modeling of geological materials: from brittle fracture to ductile flow. Comput. Methods Appl. Mech. Eng. 330, 1-32 (2018)

2. Na, S., Sun, W.C.: Computational thermo-hydro-mechanics for multiphase freezing and thawing porous media in the finite deformation range. Comput. Methods Appl. Mech. Eng. 318, 667-700 (2017)

3. Na, S., Sun, W.C.: Computational thermomechanics of crystalline rock. Part I: a combined multi-phase-field/crystal plasticity approach for single crystal simulations. Comput. Methods Appl. Mech. Eng. 338, 657-691 (2018)

4. Na, S., Sun, W.C., Yoon, H., Ingraham, M.: Effects of elastic heterogeneity on the fracture pattern and macroscopic effective toughness of Mancos Shale in Brazilian tests. J. Geophys. Res. Solid Earth 122(8), 6202-6230 (2017)

5. Ulven, O.I., Sun, W.C.: Capturing the two-way hydro-mechanical coupling effect on fluiddriven fracture in a dual-graph lattice beam model. Int. J. Num. Anal. Methods Geomech. 42(5), 736-767 (2018)

6. Wang, K., Sun, W.C.: A unified variational Eigen-erosion framework for interacting fractures and compaction bands in brittle porous media. Comput. Methods Appl. Mech. Eng. 318, 1-32 (2017)

7. Wang, K., Sun, W.C.: A multiscale multi-permeability poroplasticity model linked by recursive homogenizations and deep learning. Comput. Methods Appl. Mech. Eng. 334(1), 337-380 (2018) 